\title{
Compressible Stokes Flow in Thin Films
}

\section{E. A. van Odyck \\ C. H. Venner \\ University of Twente, \\ Faculty of Mechanical Engineering, \\ Tribology Group, \\ P.0. Box 217, \\ 7500 AE Enschede, \\ The Netherlands}

A multigrid numerical solution algorithm has been developed for the laminar (Stokes) flow of a compressible medium in a thin film. The solver has been applied to two model problems each representative of lubrication problems in a specific way. For both problems the solutions of the Stokes equations are compared with the solutions of the Reynolds equation. The configurations of both model problems were chosen such that based on the ratio film thickness to contact length $(H / L)$ the difference between the Reynolds and the Stokes solutions will be very small, so the geometry of the gap itself does not lead to a significant cross film dependence of the pressure. It is shown that in this situation the compressibility can still lead to a cross-film pressure dependence which is predicted by the Stokes solution and not by the Reynolds solution. The results demonstrate that limitations exist to the validity of the Reynolds equation related to the compressibility of the medium. [DOI: $10.1115 / 1.1539058]$

\section{Introduction}

In hydrodynamic (or aerodynamic) lubrication theory it is common practice to use the Reynolds equation [1], to describe the flow in the gap between the surfaces of the "contacting" machine elements. This equation is valid under the assumption that the ratio of film thickness to contact length is small and that the Reynolds number is also small. If the Reynolds number is too large (high speed applications) inertia effects have to be taken into account. If the ratio of film thickness to contact length is too large the Stokes equations have to be solved instead of the Reynolds equation. If both numbers are not small then the full Navier Stokes equations must be solved. Based on the nominal film geometry in most tribological settings these numbers are typically $O(0.01)$, and there seems little reason to doubt the validity of the solutions to the Reynolds equation. However, when looking at the micro geometry of the surface (surface roughness or waviness) the local values of the ratio film thickness to roughness wavelength may be significantly larger. In such cases, at least locally, the Reynolds equation may not be accurate, and the Stokes equations should be used to model the flow.

The limitation of the validity of Reynolds equation related to the film aspect ratio can be referred to as a geometric limitation. In the past, several studies have been performed to study this limitation in relation to the effect of surface roughness, see Sun [2], Phan-Thien [3] and Myllerup [4,5]. Most of these studies are analytical. So far only a few authors Noordmans [6], Schäfer [7], Odyck $[8,9]$ have actually (numerically) solved the Stokes equations for tribologically relevant problems.

Recently Bair [10] pointed out that there is another limitation to the validity of the Reynolds equation; namely, the pressure dependence of the viscosity, and of the density of the lubricant. In the standard derivation of the Reynolds equation when introducing the narrow gap assumption, the effect of this dependence on the magnitude of the different terms is not taken into account. Only after the nondimensionalization and cancellation of terms that are small on the basis of geometrical arguments is the dependence of the viscosity and density with pressure reintroduced. In view of the exponential viscosity-pressure dependence, the increase of the viscosity with pressure may be so large that terms which are neglected using geometrical arguments may not be small when the actual magnitude of the viscosity gradients would be accounted for. The same may be true if the density strongly depends on the

Contributed by the Tribology Division for publication in the ASME JOURNAL OF TRIBOLOGY. Manuscript received by the Tribology Division January 8, 2002; revised manuscript received October 22, 2002. Associate Editor: L. San Andrés. pressure as is the case for gas lubrication. So, the viscosity pressure dependence and the compressibility can lead to cross-film pressure variations which can not be predicted with models based on the Reynolds equation. The upshot of this paper is to show that such effects can indeed occur in a compressible flow.

Two compressible flow model problems are studied. The first problem has a direct relation to applications in grooved bearings. The second problem is of interest for its relation to conventional hydrodynamic lubrication. For both problems differences between the Stokes and the Reynolds solutions induced by the compressibility behavior are shown.

\section{Theory}

The objective is to study differences between the Stokes and the Reynolds solutions for the problems considered. The Stokes solutions are supposed to be the more accurate ones. Below the basic equations are presented. It is noted that only two dimensional steady state problems are considered.

The Stokes equations follow from the Navier-Stokes equations when it is assumed that inertia effects can be neglected, i.e. when the Reynolds number is small, as is explained in most textbooks on fluid dynamics, e.g. Batchelor [11] or Langlois [12]. After substitution of the following dimensionless variables in the Navier Stokes equations:

$$
\begin{aligned}
& u \rightarrow u_{c} \bar{u}, \\
& w \rightarrow \epsilon u_{c} \bar{w}, \quad \epsilon=\frac{H}{L}, \\
& x \rightarrow L \bar{x}, \\
& z \rightarrow H \bar{z}, \\
& \rho \rightarrow \rho_{c} \bar{\rho}, \\
& \eta \rightarrow \eta_{c} \bar{\eta}, \\
& p \rightarrow \frac{\eta_{c} u_{c} L}{H^{2}} \bar{p} .
\end{aligned}
$$

see Myllerup [5], where $u_{c}$ is a characteristic speed, $L$ a characteristic length in the $x$-direction, $H$ a characteristic height in the $z$-direction (nominal film height), $\rho_{c}$ a characteristic density, and $\eta_{c}$ a characteristic viscosity and, assuming that the Reynolds number based on the film height: 


$$
\operatorname{Re}=\frac{\rho_{c} u_{c} H}{\eta_{c}}
$$

is small and neglecting the terms multiplied with this number, the momentum equations for the steady state two dimensional problem are:

$$
\begin{gathered}
\epsilon^{2} \frac{\partial}{\partial x}\left[\frac{2}{3} \eta \frac{\partial w}{\partial z}-\frac{4}{3} \eta \frac{\partial u}{\partial x}\right]-\frac{\partial}{\partial z}\left[\epsilon^{2} \eta \frac{\partial w}{\partial x}+\eta \frac{\partial u}{\partial z}\right]=-\frac{\partial p}{\partial x}, \\
\epsilon^{2} \frac{\partial}{\partial z}\left[\frac{2}{3} \eta \frac{\partial u}{\partial x}-\frac{4}{3} \eta \frac{\partial w}{\partial z}\right]-\frac{\partial}{\partial x}\left[\epsilon^{2} \eta \frac{\partial u}{\partial z}+\epsilon^{4} \eta \frac{\partial w}{\partial x}\right]=-\frac{\partial p}{\partial z},
\end{gathered}
$$

and the equation of continuity is given by:

$$
\frac{\partial(\rho u)}{\partial x}+\frac{\partial(\rho w)}{\partial z}=0 .
$$

Where, for convenience of notation the bars on the scaled quantities have been dropped. Equations (3-5) will be referred to as the "Stokes model." The domain on which they are solved and the boundary conditions that will be assumed are explained in the sections describing the problems in detail. It should be noted that in all cases presented here, the upper surface is stationary, and the flat lower surface is moving in the $x$-direction. The two important parameters in the problems considered are then the film aspect ratio $(\epsilon=H / L)$ and the nondimensional lower surface speed $\left(u_{s}\right)$. The effects of $\epsilon$ on the solution have been described in detail in Odyck [8,9].

In this paper the lower surface speed $u_{s}$, is the important parameter to study. It should be noted that it is not a just a scaling parameter as the equations are now nonlinear.

For small $\epsilon$ the Stokes equations simplify to the Reynolds equation

$$
\frac{d}{d x}\left[-\rho h^{3} \frac{d p}{d x}+6 \rho h u_{s}\right]=0
$$

and in this case only one parameter remains: $u_{s}$ the (dimensionless) lower surface speed.

To compare the Stokes and the Reynolds solutions to a problem, the generated load force can be used. For the Reynolds solution this load force consists only of the integral over the pressure. For the Stokes case a velocity gradient also appears in the load force which is then defined by:

$$
F=F_{p}+F_{w}=\int_{-1}^{1} p(x, 0) d x-\left.\int_{-1}^{1} \frac{4}{3} \epsilon^{2} \frac{\partial w}{\partial z}\right|_{z=0} d x
$$

The $F_{w}$ term in Eq. (7) is associated with the normal stress on the lower surface and it drops in the pure Reynolds' case.

\section{Problem Description}

In the previous section the basic equations that will be used to model the flow were out-lined. These equations will be used to solve the two "model" problems that are described in the following sections.

3.1 Gas Lubrication Problem. As a first model problem the surface driven flow of a gas through a restriction is treated, see Fig. 1. It can be thought as a section taken from a "grooved" bearing. The lower surface moves with a certain velocity. Of particular interest is the generated load bearing capacity in relation to the surface speed and the geometry of the restriction. Because of the compressibility of the gas, according to the Reynolds based models, this load bearing capacity has a maximum that is independent of speed, see Constantinescu [13]. The boundary conditions are given in the figure.

It is common in gas lubrication to take the viscosity of the gas to be constant and for the density pressure relation to use the ideal gas law:

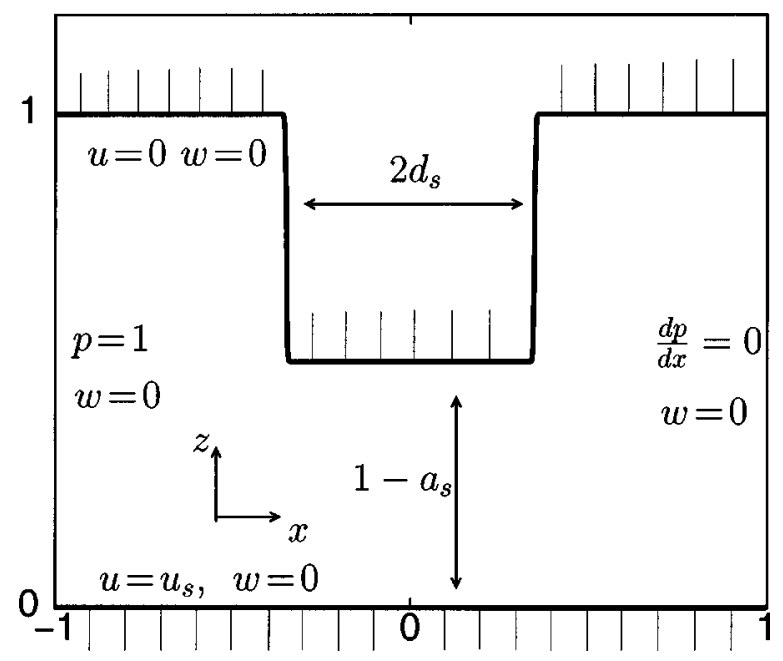

Fig. 1 Geometry and boundary conditions for gas bearing model problem

$$
p=\rho R_{g} T,
$$

where $R_{g}$ is the gas constant and the temperature $T$ has been assumed constant.

The fluid motion can be described by the Stokes equations (3), (4), and (5) or by the Reynolds equation (6). The solutions to the two equations will be compared. The density pressure dependence according to the ideal gas law does not lead to extra parameters as the proportionality constant appears in each term of the fluid motion equations so it cancels out. As mentioned previously the viscosity is assumed to be constant $(\eta=1)$.

The geometry of the restriction is defined by:

$$
h(x)=1-\frac{a_{s}}{\left(1-e^{-\kappa_{s}\left(d_{s}-x\right)}\right)\left(1+e^{-\kappa_{s}\left(d_{s}+x\right)}\right)} .
$$

where in this work $a_{s}(=0.5)$ and $d_{s}(=0.05)$ are fixed, and the parameter $\kappa_{s}$ is varied. This parameter $\kappa_{s}$ is proportional to the slope of the restriction because:

$$
\left.\frac{d h}{d x}\right|_{x=d_{s}}=\tan (\alpha) \approx \frac{a_{s} \kappa_{s}}{4}
$$

if $\kappa_{s} d_{s} \gg 1$

The pressure is set to unity at the in and outflow boundaries. In that case the solutions of the Stokes equations for different velocities of the lower surface are independent, so the flow field depends on the two parameters $\epsilon$ and $u_{s}$, unlike the solution to the Reynolds equation for the same problem which depends only on $u_{s}$. In this study the value of $\epsilon(=0.005)$ is fixed. This is a small value for which one would normally expect the Reynolds equation to be accurate. The remaining input parameters to be varied are the surface velocity $\left(u_{s}\right)$ and the geometry parameter $\left(\kappa_{s}\right)$.

In Tribology it is common to use the bearing number $\Lambda$ to characterize the condition of a gas lubrication problem. This (dimensionless) number appears in Eq. (6) when the pressure is scaled on the atmospheric pressure $p_{a}$ instead of the dimensionless pressure used here and $\eta_{c}=\eta\left(p_{a}\right)$ is used:

$$
\Lambda=\frac{6 \eta_{c} L u_{s} u_{c}}{p_{a} H^{2}}
$$

where $u_{s}$ denotes the (dimensionless) runner velocity as in Eq. (6), so the product $u_{s} u_{c}$ is simply the actual velocity.

Thus, apart from a multiplicative constant, results shown in terms of variation of $u_{s}$, can directly be translated to variation of the bearing number. The parameter of interest is the load force. 


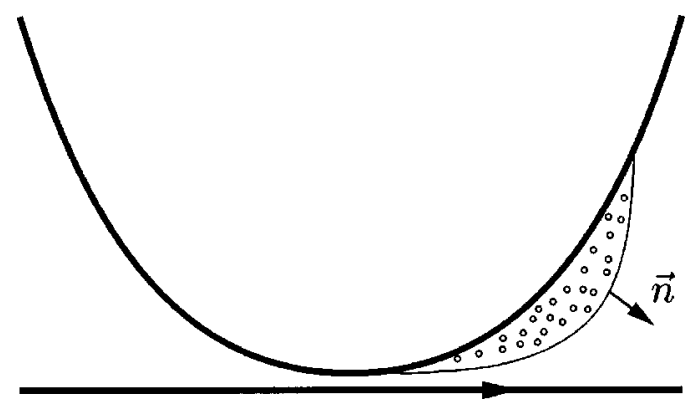

Fig. 2 Cavitation bubble in the downstream portion of the contact

For the Stokes case this force has two components $F_{p}$ and $F_{w}$, see Eq. (7). The load force according to the Reynolds solution is defined as:

$$
F_{\text {rey }}=\int_{-1}^{1} p(x) d x
$$

As mentioned previously, according to Reynolds models, gas bearings have a limited maximum load bearing capacity. For the present problem this high speed limit can be calculated as follows. Dividing Eq. (6) by $u_{s}$, integrating it with respect to $x$, taking limit $u_{s} \rightarrow \infty$ and, using the fact that $\rho$ is proportional to $p$, one obtains:

$$
6 p h=c_{1},
$$

where the integration constant $c_{1}$ follows from the pressure boundary condition $6 p(-1) h(-1)=6=c_{1}$. In the high-speed limit the load force can be calculated according to:

$$
F_{\text {rey }}^{\infty}=\int_{-1}^{1} \frac{1}{h} d x .
$$

So, for a given geometry this limit can be computed easily. When $\kappa_{s} \rightarrow \infty$, representing exactly a step, the integral can be evaluated:

$$
F_{\text {rey }}^{\infty}=2+\frac{2 a_{s} d_{s}}{\left(1-a_{s}\right)}
$$

which for $a_{s}=0.5$ and $d_{s}=0.05$ gives $F_{\text {rey }}^{\infty}=2.1$. This straightforwardly follows from integration of the pressure profile given by $p=1 / h$ and Eq. (9).

3.2 Fluid Lubrication With Cavitation. The second problem model problem is the flow of a liquid below a parabola which is a classic hydrodynamic lubrication problem. Unlike the gas lubrication problem described in the previous section now the boundary pressure on each side will be the gauge pressure. When solving this problem using either the Stokes equations or the Reynolds equation because of the symmetric height geometry the pressure field will be antisymmetric, and the resulting net load capacity of the pressure profile will be zero. In reality a liquid can not sustain large tensile stresses and it will cavitate when the pressure drops below the vapor pressure and as a result in reality there will be a net load carrying capacity.

Figure 2 shows an illustration of the type of vapor/gas bubble observed experimentally in the flow under a stationary curved upper surface driven by the moving lower surface, see Dowson [14]. It was found to occur downstream of the narrowest gap location near the stationary surface. On the moving surface a layer of liquid remains.

To simulate cavitation behavior the flow models have to be changed. When using models based on the Reynolds equation this can be done quite straightforwardly by limiting the computed pressure from below with a complementarity condition, see Elrod

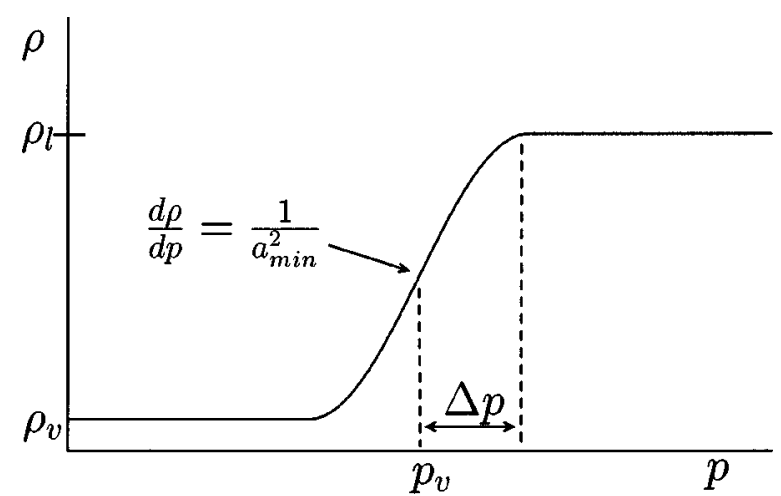

Fig. 3 Pressure-density diagram for the liquid/vapor mixture

[15]. However, this approach can not be used in combination with the Stokes equations. In this section an alternative approach is shown to simulate the pressure limiting aspect of cavitation. It has the advantage that it can be used in both Stokes and Reynolds based models. In fact for Reynolds based models it is even easier to implement than the aforementioned complementarity approach. The upshot is to treat the problem as a two phase (TP) flow problem or, the flow of a compressible medium with a special type of density pressure relation. The model used here is based on the work of Delannoy [16] and Hoeijmakers [17]. No distinction needs to be made between the cavitated region and the pressurized region. Consequently it is easy to implement in a computational method. It may not correctly describe the physical mechanisms inside the cavity or at its boundary, but it will at least adequately prevent the pressure from dropping below the vapor pressure and indicate the location and size of the cavitated region.

It is assumed that the vapor and the liquid move at the same speed, otherwise for each state separate equations would be needed with interaction terms. In modeling the problem it is further justified to take the saturation pressure and the vapor pressure both equal to the ambient pressure which is set to the gauge pressure (zero) as is usually done in lubrication problems. In the TP model the dependent variables refer to the mixture and an equation of state is used to complete the system:

$$
\rho=\left\{\begin{array}{cl}
\rho_{l} & \text { if } p>p_{v}+\Delta p, \\
\rho_{v} & \text { if } p<p_{v}-\Delta p, \\
\rho_{v}+\Delta \rho\left[1+\sin \left(\frac{p-p_{v}}{\Delta \rho a_{\mathrm{min}}^{2}}\right)\right] & \text { otherwise }
\end{array}\right.
$$

where

$$
\begin{aligned}
p_{v} & =\text { vapor pressure } \\
\rho_{l} & =\text { density liquid } \\
\rho_{v} & =\text { density vapor } \\
\Delta \rho & =\frac{1}{2}\left(\rho_{l}-\rho_{v}\right) \\
\Delta p & =\frac{1}{2} \pi a_{\min }^{2} \Delta \rho \\
a_{\text {min }}=\text { minimal } & \text { speed of sound in mixture }
\end{aligned}
$$

Figure 3 gives a graphical representation of $\rho(p)$. The parameter $a_{\text {min }}$ can be approximated by $a_{\text {min }} \approx 2 a_{v} \sqrt{\rho_{v} / \rho_{l}}$ if $\rho_{l} \gg \rho_{v}$ with $a_{v}$ the speed of sound in the vapor (see Appendix). For water at standard conditions $a_{\text {min }} \approx 25[\mathrm{~m} / \mathrm{s}]$. After scaling according to Eq. (1), with $\rho_{c}=\rho_{l}$, one obtains: 


$$
\bar{\rho}=\left\{\begin{array}{cc}
1 & \text { if } \bar{p}>\bar{p}_{v}+\frac{\pi}{2 \beta_{\rho}}, \\
\bar{\rho}_{c} & \text { if } \bar{p}<\bar{p}_{v}-\frac{\pi}{2 \beta_{\rho}}, \\
\bar{\rho}_{c}+\frac{1}{2}\left(1-\bar{\rho}_{c}\right)\left[1+\sin \left(\beta_{\rho}\left(\bar{p}-\bar{p}_{v}\right)\right)\right] & \text { if } \bar{p}_{v}-\frac{\pi}{2 \beta_{\rho}} \leqslant \bar{p} \leqslant \bar{p}_{v}+\frac{\pi}{2 \beta_{\rho}}
\end{array},\right.
$$

where

$$
\begin{aligned}
& \bar{\rho}_{c}=\frac{\rho_{v}}{\rho_{l}}, \\
& \beta_{\rho}=\frac{\eta_{c} u_{c} L}{H^{2} \Delta \rho a_{\min }^{2}}, \\
& \bar{p}_{v}=\frac{p_{v} H^{2}}{\eta_{c} u_{c} L} .
\end{aligned}
$$

For convenience the bars on the scaled quantities will be dropped. Next, for the correct relation between viscosity and pressure when the pressure approaches the vapor pressure there are different options, see Kubota [18]. For example, a constant viscosity or a viscosity that exhibits the same behavior as the density as a function of the pressure, i.e., Eq. (16) with $\eta_{l}$ replacing $\rho_{l}$ and $\eta_{v}$ replacing $\rho_{v}$.

The model problem considered in this paper is now formed by the computation of the flow under a parabolically shaped surface given by:

$$
h(x)=0.8 x^{2}+0.2
$$

using the Stokes equations, Eq. (3-5), and the two-phase densitypressure Eq. (18). The viscosity is assumed to be constant. The boundary conditions are $p=0, w=0$ at the inlet and at the outlet. The parameter to be varied is the parameter $\beta_{\rho}$ which represents the gradient of the density pressure curve in the transition region.

\section{Numerical Solution}

The approach to obtain numerical solutions is in principle the same as for the incompressible case, see Odyck [8,9]. Equations (3-5) have been transformed to curvi-linear coordinates using height scaling. The transformed domain is rectangular. On this domain the transformed equations have been discretized using a conservative discretization and a staggered grid arrangement. The discrete system of equations is solved by means of a Multigrid algorithm built around a Symmetric Coupled Gauss-Seidel relaxation.

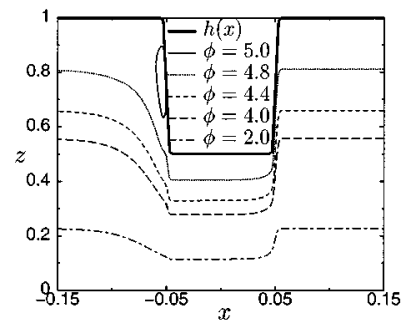

(a)

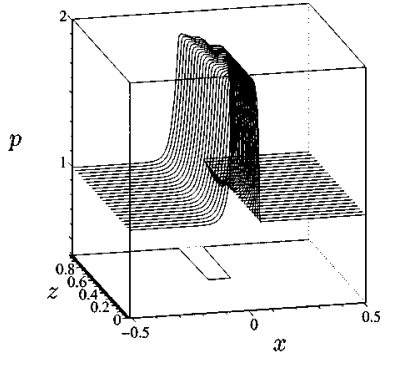

(b)
Fig. 4 Stokes solution: (a) streamlines; and (b) pressure field. Both as function of $x$ and $z$ for $\epsilon=0.005, u_{s}=10.0$ and $\kappa_{s}$ $=1343$.
To have good efficiency for small values of the domain aspect ratio $H / L$ it is required that this relaxation be carried out as a $z$-line relaxation. The main difference with the incompressible problem discussed in Odyck $[8,9]$ is the discretization of the equation of continuity which has been changed from a second order central to a first order upwind discretization. This was done for stability reasons as the compressible problem has a hyperbolic character. Because the discretization is first order the solutions will tend to be less accurate and should carefully be checked for effects of "artificial diffusivity (viscosity)." This was done by comparing solutions obtained on different grids. For specific details regarding the discretization and the numerical algorithm the reader is referred to Odyck $[8,9]$.

The solutions presented in the following section were all obtained using a Full Multi-Grid algorithm with $5 \mathrm{~V}(2,2)$ cycles per grid level. The finest grid contained $256 \times 256$ cells. The error reduction per Multigrid cycle was grid independent. For the gas lubrication problem an error reduction per cycle of an order of magnitude was easily obtained. Moreover, the larger the runner velocity in the problem, the higher the speed of convergence. This could be expected and is related to the choice of an upstream discretization combined with $z$-line relaxation. For the cavitation problem the convergence rate rather strongly depended on the value of the parameter $\beta_{\rho}$ of the density pressure relation. A backtracking algorithm in the line relaxation was needed to ensure convergence for the larger values of $\beta_{\rho}$. In all cases presented in this paper with the aforementioned number of cycles the error on the target grid was reduced to a level much smaller than the discretization error.

\section{Results}

In this section the results obtained for the two model problems are presented and discussed. In particular attention will be given to the differences between the solutions of the Stokes model and the solutions of the Reynolds equation in relation to the problem parameters. In each section also some results are given to illustrate the accuracy of the solutions.

5.1 Gas Lubrication Problem. Figure 4 shows a typical solution to the problem for the case $\kappa_{s}=1343$ and $u_{s}=10.0$. For this value of $\kappa_{s}$ the actual angle between the restriction and the

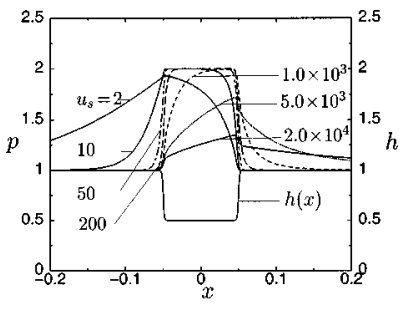

(a)

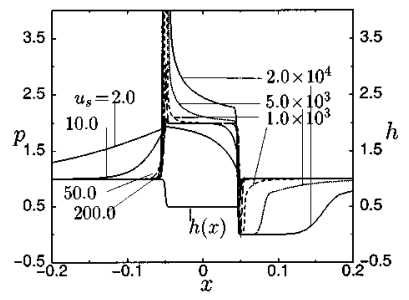

(b)
Fig. 5 Pressure as function of $x$ for $\epsilon=0.005, \kappa_{s}=1343$ and different $u_{s}$ for the Stokes solution to the gas bearing problem. (a) Pressure at $z=0$; and (b) pressure at $z=h$. In (a) the Reynolds solution overlaps the Stokes solution for $u_{s}=2,10,50$. 
Table 1 Computed load force for gas lubrication problem for Reynolds solution $\left(F_{\text {rey }}\right)$ Stokes solution $\left(F_{p}, F_{w}\right)$ as a function of the lower surface speed $u_{s}$. The Reynolds limit for infinite $u_{s}$ is $F_{\text {rey }}^{\infty}=2.1$.

\begin{tabular}{lcccc}
\hline \hline & \multicolumn{3}{c}{ Stokes } & \multirow{2}{*}{$\begin{array}{c}\text { Reynolds } \\
u_{s}\end{array}$} \\
\cline { 2 - 4 } & $F_{p}$ & $F_{w}$ & $F_{p}+F_{w}$ & \\
\hline 2 & 2.1947 & 0 & 2.1947 & 2.1802 \\
10 & 2.1245 & 0 & 2.1245 & 2.1157 \\
50 & 2.1101 & 0 & 2.1101 & 2.1015 \\
200 & 2.1084 & 0 & 2.1084 & 2.0994 \\
$1.0 \times 10^{3}$ & 2.1081 & 0.0003 & 2.1084 & 2.0991 \\
$5.0 \times 10^{3}$ & 2.1072 & 0.0010 & 2.1082 & 2.0990 \\
$2.0 \times 10^{4}$ & 2.0892 & 0.0102 & 2.0994 & 2.0990 \\
\hline \hline
\end{tabular}

horizontal is $40 \mathrm{deg}$. The figure shows the streamlines and a the pressure field as a function of $x$ and $z$. The streamline figure shows that the major part of the flow passes under the restriction but that a small recirculation zone occurs at its leading edge. The recirculation is also predicted by the solution to the Reynolds equation for this case. The pressure field shows no unexpected features: a "block-shaped" pressure distribution along the restriction with a "transition region" on its leading and trailing edge in which the pressure builds up to the high level and decreases again from this level. Based on the Reynolds solution one would now expect the width of this transition region to decrease with increasing speed. However, the solutions to the Stokes equations show a different behavior. Figure 5 shows the computed pressure profiles at $z=0$ and $z=h$ as a function of $u_{s}$ for the Reynolds and the Stokes solutions to the problem. The Reynolds solutions with increasing speed show the aforementioned behavior. According to the Stokes solutions initially with increasing $u_{s}$ indeed a block shape appears, and for low speeds the pressure profiles at $z=0$ and $z=h$ differ very little which indicates no cross-film pressure dependence. However, with increasing $u_{s}$ the pressure profile moves away from the block shape and at the same time the profile at $z$ $=0$ and that at $z=h$ start to differ. For $z=0$ the high pressure at the restriction followed by a low pressure in its wake is replaced by a gradual increase along the restriction and a gradual decrease in its wake. At the surface $z=h$ the pressure profile tends to exhibit a narrow peak at the leading edge of the restriction and also here along the restriction it is not constant but shows a gradual decrease. In the wake of the restriction $(x>0.05)$ a zone of very low pressure is formed (nearly vacuum). The length of this zone increases with increasing $u_{s}$.

Summarizing it is concluded that with increasing speed one observes that a cross-film pressure dependence develops related to the compressibility of the medium. This cross-film dependence is naturally not predicted by the Reynolds solution to the problem and is the type of limitation to the Reynolds equation that was indicated by Bair et al. [10].

Having observed that the Stokes and Reynolds solution for high speeds are very different the question arises if the maximum load

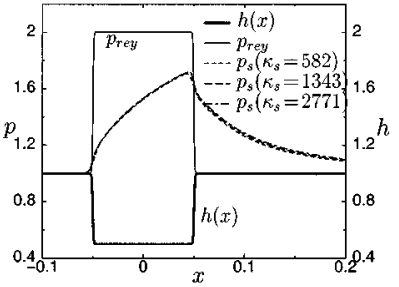

(a)

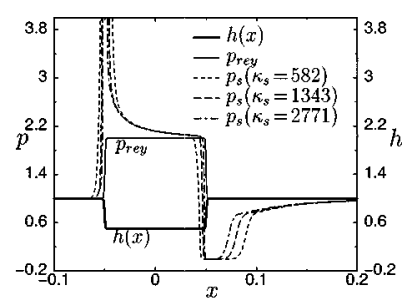

(b)
Fig. 6 Pressure as function of $x$ for $\epsilon=0.005, u_{s}=5.0 \times 10^{3}$ and different $\kappa_{s}$ for the Stokes $\left(p_{s}\right)$ and Reynolds $\left(p_{\text {rey }}\right)$ solutions to the gas bearing problem: (a) pressure at $z=0$; and (b) pressure at $z=h$

capacity of the configuration as it is predicted from the Reynolds solution is accurate. Table 1 shows the computed load capacity of the configuration as a function of $u_{s}$ for the Stokes and Reynolds solutions. The table shows that the computed load forces for the two solutions differ little and, quite remarkably, in spite of the fact that the Stokes solution to the pressure does not seem to have a high-speed limit, the load force does. Moreover, its limiting value is the same as the one predicted by the Reynolds model. Thus, for bearing design in terms of load carrying capacity the observed differences between the Reynolds solution and the Stokes solutions are not likely to have significant consequences. However, they may be important for operational aspects related to local phenomena such as surface damage by fatigue which can lead to failure.

The effect of the slope of the side wall of the restriction is the next topic of investigation. The parameter in the model representative of this slope is $\kappa_{s}$, see Eq. (10). In Fig. 6 results are presented for a given (large) $u_{s}$ and three values of $\kappa_{s}: \kappa_{s}=582$ which coincides with an angle $(\alpha=20 \mathrm{deg}), \quad \kappa_{s}=1343(\alpha$ $=40 \mathrm{deg})$ and $\kappa_{s}=2771(\alpha=60 \mathrm{deg})$. The figure shows that the pressure on the lower surface is hardly effected by a change of $\kappa_{s}$. However, the pressure on the upper surface does change. The width of the pressure peak preceding the step decreases with increasing $\kappa_{s}$ and so does the length of the nearly vacuum zone following the restriction. So, it appears that if the lower surface speed is fixed and the slope of the side walls is increased, the pressure along the upper surface will approximate the Reynolds high speed limit of $p=1 / h$, except for a pressure peak before the step, and a suction dip behind the step. The lower surface pressure profile on the other hand is hardly affected by a change in $\kappa_{s}$ but it is totally different from the high-speed Reynolds limit solution. Finally, Table 2 gives the computed load force according to the Stokes and Reynolds solution for the different values of $\kappa_{s}$ and velocities $u_{s}=2.0$ and $u_{s}=5.0 \times 10^{3}$, respectively. In this table it can be seen that the load capacity does not strongly depend on the value of $\kappa_{s}$ and again its value computed using the Stokes solution differs little from the value obtained from the Reynolds solution.

To conclude this section, some details are given regarding the

Table 2 Computed load force for gas lubrication problem for Reynolds solution ( $\left.F_{\text {rey }}\right)$ Stokes solution $\left(F_{p}, F_{w}\right)$ as a function of the step parameter $\kappa_{s}$. The Reynolds limit for infinite $u_{s}$ is $F_{\text {rey }}^{\infty}=2.1$.

\begin{tabular}{llllc}
\hline \hline & & & \multicolumn{2}{c}{ Stokes } \\
\cline { 3 - 4 }$u_{s}$ & $\kappa_{s}$ & $F_{p}$ & $F_{w}$ & $F_{p}+F_{w}$ \\
\hline 2.0 & 582 & 2.1894 & 0 & 2.1894 \\
2.0 & 1343 & 2.1947 & 0 & 2.1947 \\
2.0 & 2771 & 2.1970 & 0 & 2.1970 \\
$5.0 \times 10^{3}$ & 582 & 2.1046 & 0.0005 & 2.1051 \\
$5.0 \times 10^{3}$ & 1343 & 2.1072 & 0.0010 & 2.1082 \\
$5.0 \times 10^{3}$ & 2771 & 2.1090 & 0.0013 & 2.0976 \\
\hline \hline
\end{tabular}




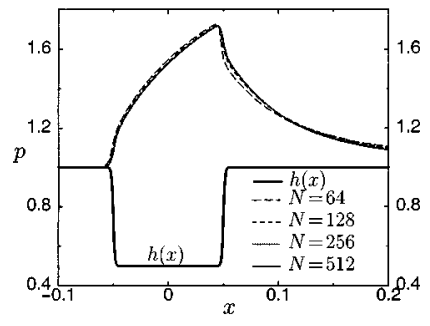

(a)

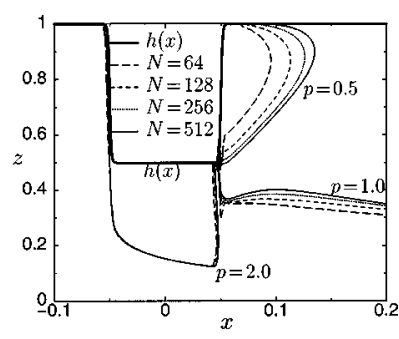

(b)
Fig. 7 (a) Pressure as a function of $x$ at $z=0$ for the Stokes solution on different grids; and (b) contour plot of the pressure for the Stokes solution on different grids. $\epsilon=0.005, u_{s}=5.0$ $\times 10^{3}$ and $\kappa_{s}=1343$.

accuracy of the solutions. Because a first-order upstream discretization was used, it is important to check whether observed tendencies in the solution are not a result of artificial "viscosity." This applies in particular to the solutions for large $u_{s}$. Convergence of the solution with decreasing mesh size was checked carefully and some results are shown in Fig. 7. This figure shows the pressure as a function of $x$ at $z=0$ according to the Stokes solution for grids with $N=64,128,256,512$. Also shown is a plot of some of the contour lines of the pressure. The figure shows that with decreasing mesh size the solution converges. This process for the pressure contours is quite slow, as could be expected from the use of a first order scheme. However, the computed pressure profiles are sufficiently accurate to be able to conclude that the differences between the Stokes solution and the Reynolds solution observed for large $u_{s}$ are "real" and not due to artifacts of the discretization on a grid that is too coarse.

5.2 Fluid Lubrication Problem With Cavitation. For this problem results are presented obtained for values of the parameter $\beta_{\rho}$ of the cavitation model in the range $10 \leqslant \beta_{\rho} \leqslant 4.0 \times 10^{4} . \beta_{\rho}$ represents the slope of the density-pressure curve around the vapor pressure point. A larger value implies a steeper slope and is likely to have an effect on the solution in and near the cavitation region. A typical solution to the problem is shown in the Figs. 8 and 9. The results are obtained for $\epsilon=0.01, p_{v}=-3.0$ and $\beta_{\rho}$ $=10$. In Fig. 8 are shown the pressure profile and the streamlines as a function of $x$ and $z$. From the figure it can be seen that the two-phase model indeed leads to the desired behavior. The pressure is bounded below by the vapor pressure. This would not have been the case without the model as is illustrated in Fig. $9(a)$ where, for the same case are shown the pressure at $z=0$ computed using the Reynolds model with and without cavitation, and the present solution of the Stokes model with cavitation accounted for. From the figure it can be seen that for the value $\epsilon=0.01$ and $\beta_{\rho}$ taken here the Reynolds and Stokes solutions are indistinguish-

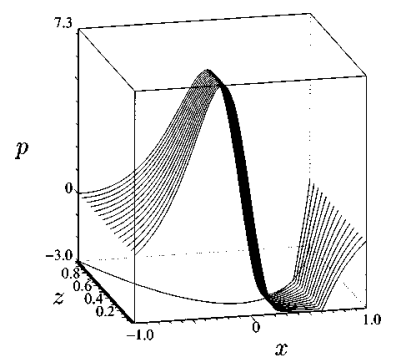

(a)

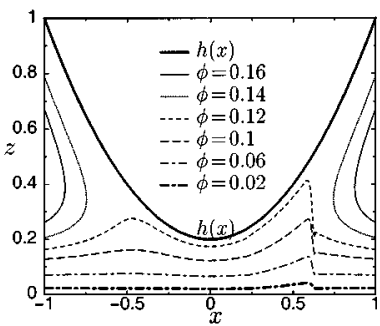

(b)
Fig. 8 (a) Pressure profile as function of $x$ and $z$; and (b) and Streamlines as function of $x$ and $z . \epsilon=0.01, \beta_{\rho}=10.0, p_{v}$ $=-3.0$.

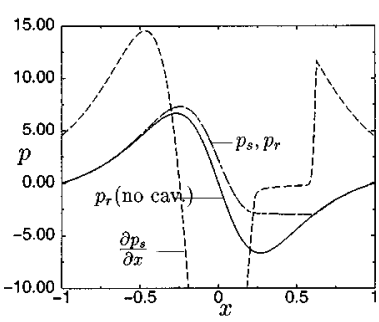

(a)

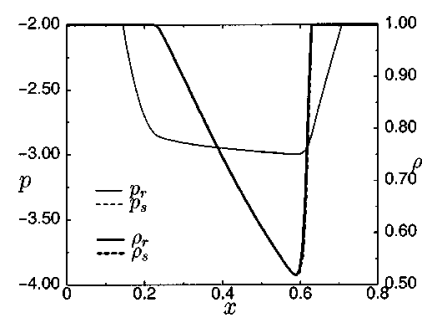

(b)

Fig. 9 (a) The pressure at $z=0$ as function of $x$ for the Reynolds solution without cavitation ( $p_{r}$ (no cav.)), the Reynolds solution with cavitation $\left(p_{r}\right)$ and the Stokes solution with cavitation $\left(p_{s}\right)$; and $(b)$ enlargement of the pressure and density at $z=0$ as function of $x$ for the Reynolds and the Stokes solution with cavitation in the cavitation region, $\epsilon=0.01, p_{v}=-3.0$, and $\beta_{\rho}=10.0$. The Reynolds and the Stokes solution with cavitation overlap in both figures.

able. As for this value of $\epsilon$ there is no cross film dependence of the pressure (see also Fig. 10(a)) it is sufficient to only show the pressure at the lower surface. When cavitation is not accounted for the solution is anti-symmetric and when it is accounted for the pressure is limited to the vapor pressure. The width of the cavitated region will depend on the value of the vapor pressure. For this case where $p_{v}$ is taken significantly lower than the boundary pressure one observes a pressure drop reaching the vapor pressure at about $x=0.2$ and a return to the liquid pressure at about $x$ $=0.6$. The Fig. $9(b)$ shows an enlargement of the pressure profiles in the cavitated region together with the density profile.

In Fig. $9(a)$ the pressure gradient $\partial p_{s} / \partial x$ is also given as a function of $x$. It can be seen that this gradient has a discontinuity at the edge of the pressure recovery zone, in this case at $x \approx 0.6$. Since $p_{s}=p_{r}$, for small $\epsilon$, a shock relation can be deduced from the Reynolds equation.

Let the variables in the cavitated region be indicated by the subscript 1 and in the liquid region following the pressure recovery point by the subscript 2 . Integrating the Reynolds equation with respect to $x$ across the shock using $h_{1}=h_{2}=h$ and $(d p / d x)_{1}=0$ it follows that:

$$
\left(\frac{d p}{d x}\right)_{2}=\frac{6}{h^{2}}\left(1-\frac{\rho_{1}}{\rho_{2}}\right)
$$

which is the equivalent of the usual Jakobsson-Floberg-Olsson (JFO) relation $[19,20]$.

In the Figs. 10 and 11 the contour plots are shown of the density for the cases $\beta_{\rho}=10,5.0 \times 10^{3}, 1.0 \times 10^{4}$ and $4.0 \times 10^{4}$, respectively. Comparing the lines for $\beta_{\rho}=10$ with those for $\beta_{\rho}$ $=5.0 \times 10^{3}, 1.0 \times 10^{4}$ and $4.0 \times 10^{4}$ shows that the width of the cavitated region is not changed. However, the lines of constant density inside this region are no longer straight which implies that

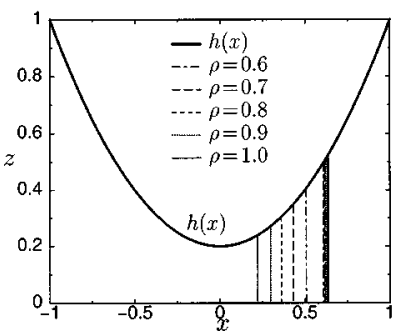

(a)

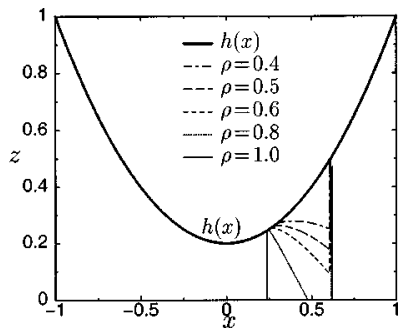

(b)

Fig. 10 Contour plot of the density for $\epsilon=0.01$ and $p_{v}=-3.0$, (a) $\beta_{\rho}=10.0$; and (b) $\beta_{\rho}=5.0 \times 10^{3}$ 


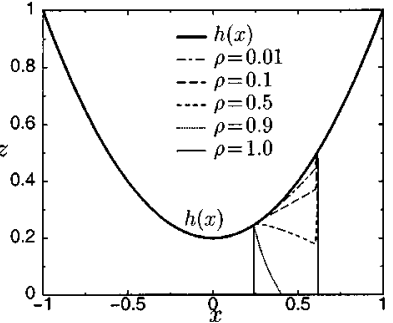

(a)

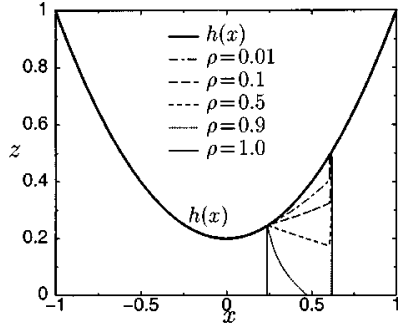

(b)
Fig. 11 Contour plot of the density for $\epsilon=0.01$ and $p_{v}=-3.0$; (a) $\beta_{\rho}=1.0 \times 10^{4}$; and (b) $\beta_{\rho}=4.0 \times 10^{4}$.

$p$ varies as a function of $z$ and that in this region the Reynolds and the Stokes solutions differ. In particular, with increasing $\beta_{\rho}$ a region of very low density develops close to the upper surface. As in this region the density equals the vapor density, this can be interpreted as a "bubble." The development of such a fully vaporized region or "bubble" with increasing $\beta_{\rho}$ is also illustrated in Fig. 12. In this figure the density is plotted as a function of $z$ at $x=0.5$ for different $\beta_{\rho}$. Note that as a function of $z$ the transition from the fully-vaporized region to the region with $\rho>0.01$ at $x$ $=0.5$ is gradual. Furthermore, it can be seen in Fig. 12 that with increasing $\beta_{\rho}$ the density near $z=0$ approaches unity, so near the lower surface the mixture tends to remain liquid. This is also what one would expect to happen from a physical point of view as for the present case the lower surface supplies the lubricant. To investigate if this formation of a fully-vaporized region is related to the pressure solution by some parameter the difference $\Delta \tilde{p}=p(x, z$ $=h(x))-p(x, z=0)$, at the point where $p$ reaches a minimum in the non-cavitating case was computed. It was found that the fully vaporized region occurs if $\Delta \widetilde{p}>\pi / 2 \beta_{\rho}$ and the minimum pressure in the non-cavitating case is lower than $p_{v}$.

Next, a few words about the influence of the ratio $\epsilon=H / L$. For the solutions shown in Figs. 10 and 11, even though the solution inside the cavitated region changes, the boundaries of this region remain straight. This can be ascribed to the small value $\epsilon$. For a larger value of $\epsilon$ these lines will no longer be straight as can be seen in Figure 13(a) where the result is shown obtained for $\epsilon$ $=0.5, p_{v}=-4.0$ and $\beta_{\rho}=20.0$. Figure 13(b) shows the density as a function of $z$ at $x=0.5$.

Having shown all this the question is if one should expect these effects to occur in practice: What value of $\beta_{\rho}$ is realistic for a mineral oil. A crude estimate can be obtained taking the following values for a mineral oil at standard conditions flowing through a hydrodynamically lubricated contact:

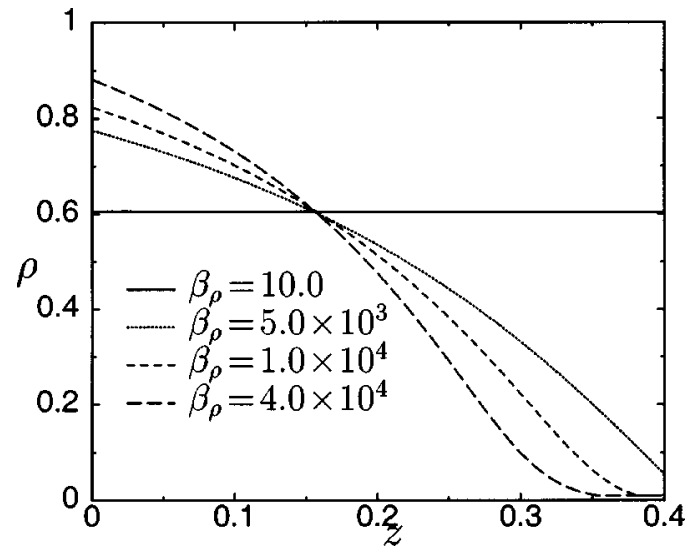

Fig. 12 Density as function of $z$ at $x=0.5$ for $\epsilon=0.01$ and $p_{v}$ $=-3.0$ (a)

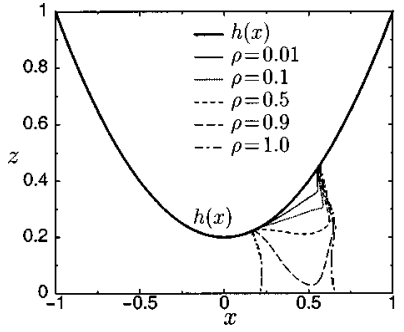

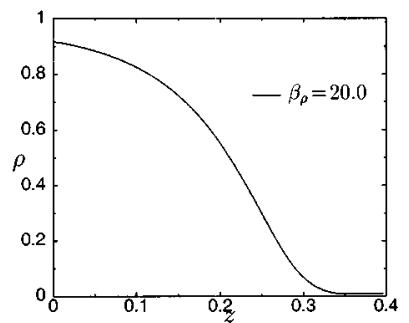

(b)
Fig. 13 (a) Contour plot of the density; and (b) density as function of $z$ at $x=0.5$. For $\epsilon=0.5, p_{v}=-4.0$ and $\beta_{\rho}=20.0$.

$$
\begin{aligned}
\eta_{o} & =1.0 \times 10^{-1} \mathrm{~kg} / \mathrm{ms} \\
\rho_{l} & =1.0 \times 10^{3} \mathrm{~kg} / \mathrm{m}^{3} \\
\rho_{v} & =1.0 \mathrm{~kg} / \mathrm{m}^{3} \\
a_{v} & =5.0 \times 10^{2} \mathrm{~m} / \mathrm{s} \\
H & =0.1 \times 10^{-6} \mathrm{~m} \\
u_{c} & =1.0 \mathrm{~m} / \mathrm{s} \\
\epsilon & =0.01
\end{aligned}
$$

From Eq. (19) it the follows that $\beta_{\rho}=20.0$. So, the $z$-dependency in the density for the problem discussed here does not seem to be unrealistic. Moreover if the nominal film thickness is smaller and/or $u_{c}$ is much larger it will only be stronger.

Summarizing, from the results presented in this section it is concluded that the cavitation model yields the desired "physical" behavior from the viewpoint of load force computations, i.e., it provides a lower limit on the pressure. The width of the cavitation region will be determined by the magnitude of the vapor pressure itself. The solution inside this region will depend on the parameter $\beta_{\rho}$. For large $\beta_{\rho}$ in this region a $z$-dependent pressure and density are found showing once again that the density-pressure relation induces differences between Stokes and Reynolds solutions in thin films.

The accuracy of the results has been verified by comparing solutions obtained on grids with a different mesh size. As an illustration, for a typical case in Fig. 14(a) the contourline associated with a given density value is shown as obtained from the solutions computed on a grid with $N=128,256$ and 512 respectively. In Fig. 14(b) is shown the density as a function of $z$ at a given location for the same solutions. The results apply to the case $\epsilon=0.01, p_{v}=-3.0$, and $\beta_{\rho}=1.0 \times 10^{4}$. These figures show that with decreasing mesh size the solution indeed converges roughly

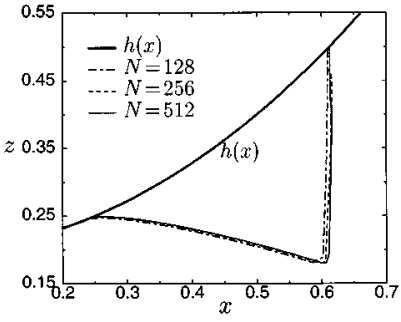

(a)

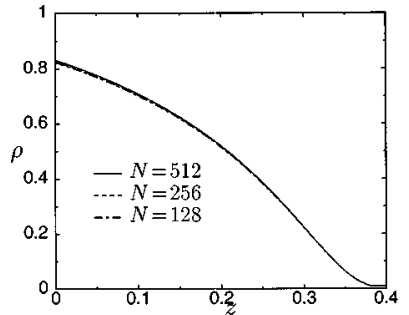

(b)
Fig. 14 (a) Contour plot of the iso-density contour $\rho=0.5$ for the Stokes solution on different grids; and (b) Density as function of $z$ at $x=0.5$ for the Stokes solution on different grids. $\epsilon$ $=0.01, p_{v}=-3.0$, and $\beta_{\rho}=1.0 \times 10^{4}$. 
first order in accordance with the first order of the discretization. Clearly the differences between the Reynolds and Stokes solutions are much larger than the error in the result.

\section{Conclusion}

A multigrid numerical solution algorithm has been developed for the laminar (Stokes) flow of a compressible medium in a thin film. The solver has been applied to two model problems representative of lubrication problems. For both problems the solutions of the Stokes equations are compared with the solutions of the Reynolds equation.

The first problem is representative of a (section in a) grooved gas bearing. It is shown that at high speeds even when according to the ratio nominal film thickness to contact length there is little reason to expect cross-film dependence of the pressure, such a dependence can occur induced by the compressibility of the gas. The pressure profile along the grooved surface resembles the Reynolds high speed limit. However, the pressure profile along the smooth surface is different. Nevertheless, the predicted maximum load force for the Stokes and the Reynolds solutions is the same.

The second problem is that of the surface driven flow of a liquid under a parabolically shaped counter-surface. This problem was selected to illustrate an alternative way to simulate cavitation behavior in hydrodynamic lubrication problems. This alternative is needed because the conventional models used with Reynolds equation are not suited to be used in combination with the Stokes equations. The alternative method considers the compressible equations combined with a special density pressure relation. It is straight-forward to implement also in models based on the Reynolds equation. It has been shown that with this approach the objective to limit the pressure from below is indeed achieved. The results compare well with the Reynolds model solutions for conditions of a small ratio nominal film thickness to contact length and when the density pressure gradient is not too large. For large density pressure gradients it is shown that the compressibility itself can lead to cross-film pressure dependences which the Reynolds equation can not predict.

\section{Acknowledgment}

This research was supported by the Dutch Technology Foundation project STW. In addition, the authors would like to thank Prof. Dr. H.W.M. Hoeijmakers for his comments and the helpful discussions.

\section{Nomenclature}

If the dimension is not stated the variable or parameter is dimensionless.

$$
\begin{aligned}
& a_{s}=\text { amplitude } \\
& a_{l}=\text { speed of sound in liquid }[\mathrm{m} / \mathrm{s}] \\
& a_{v}=\text { speed of sound in vapor }[\mathrm{m} / \mathrm{s}] \\
& a_{\text {min }}=\text { minimal speed of sound }[\mathrm{m} / \mathrm{s}] \\
& d_{s}=\text { length block in } h(x) \\
& F=\text { load force } \\
& F_{p}=\text { force perpendicular to lower surface } \\
& F_{w}= \text { force tangent to lower surface } \\
& F_{\text {rey }}= \text { force perpendicular to lower surface resulting from } \\
& \text { Reynolds solution } \\
& F_{\text {rey }}^{\infty}=\text { force perpendicular to lower surface resulting from } \\
& \text { high speed Reynolds solution } \\
& h= \text { surface geometry } \\
& H= \text { film thickness [m] } \\
& L= \text { film length }[\mathrm{m}] \\
& N= \text { number of cells on square grid in } x / z \text { direction } \\
& p=\text { pressure } \\
& p_{a}= \text { ambient pressure } \\
& p_{s}= \text { pressure of Stokes solution } \\
& p_{r}= \text { pressure of Reynolds solution } \\
& p_{v}=\text { vapor pressure [Pa] }
\end{aligned}
$$

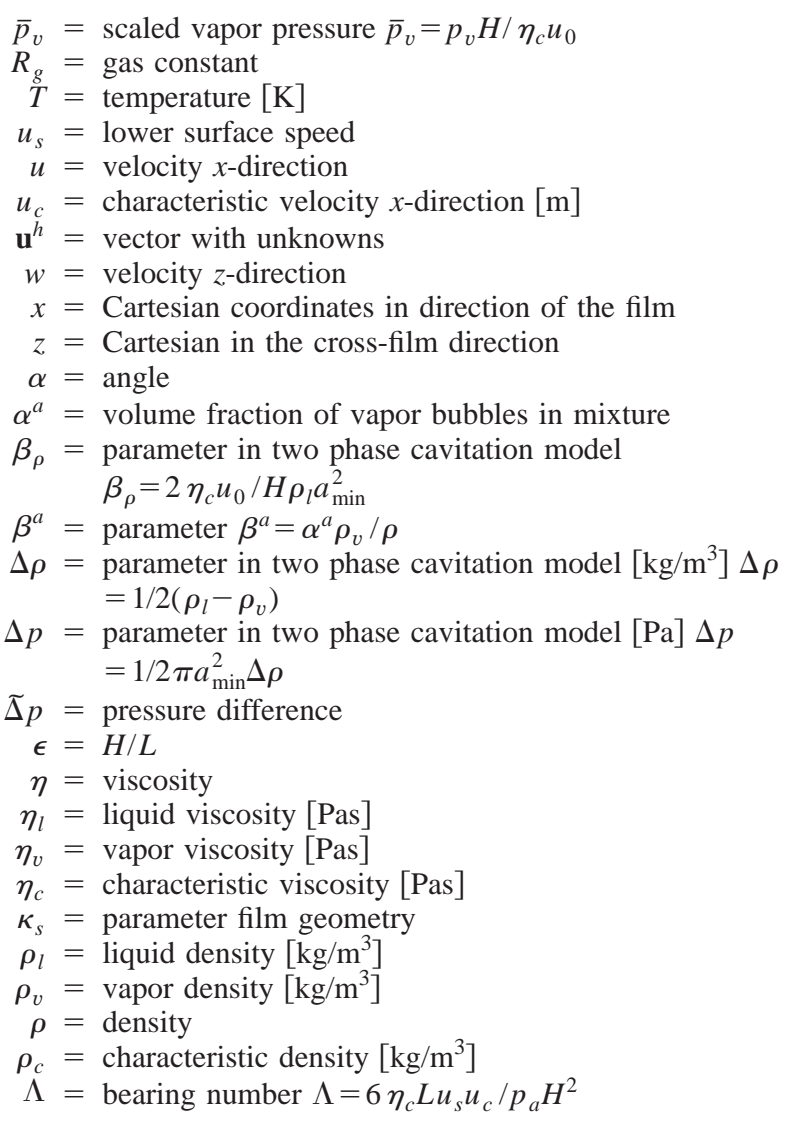

\section{Appendix A}

Determination Speed of Sound. In the two phase model the minimum speed of sound in a mixture of liquid and bubbles is required. In the following an estimate for the minimum speed of sound is deduced. For a detailed discussion see Wijngaarden [21], p. 93. This parameter is then used in Eq. (18). The speed of sound in a fluid is defined as:

$$
a^{2}=\left(\frac{\partial p}{\partial \rho}\right)_{s},
$$

where ()$_{s}$ indicates a constant entropy. Only in the transition region between the liquid and the vapor state the density is not constant and the speed of sound is not infinite (see Fig. 3). This is typical for the used model. Obviously, in reality the speeds of sound in the vapor and in the liquid have finite values.

To determine the speed of sound in the mixture the volume fraction $\left(\alpha^{a}\right)$ of the vapor bubbles in the mixture is introduced. The actual density is then a linear combination of the liquid density and the vapor density:

$$
\rho=\alpha^{a} \rho_{v}+\left(1-\alpha^{a}\right) \rho_{l} .
$$

It is assumed that the bubbles in the mixture are small enough to follow the fluid when an acoustical wave passes. In that case, the ratio $\beta^{a}=\alpha^{a} \rho_{v} / \rho$ is a conservative quantity (i.e., the mass does not change when an acoustical wave passes). It can be substituted in Eq. (24) and results in:

$$
\frac{1}{\rho}=\frac{\beta^{a}}{\rho_{v}}+\frac{1-\beta^{a}}{\rho_{l}} .
$$

This can be differentiated with respect to $p$ and after some manipulations the following equation can be found

$$
\frac{1}{a^{2} \rho}=\frac{\alpha^{a}}{a_{v}^{2} \rho_{v}}+\frac{1-\alpha^{a}}{a_{l}^{2} \rho_{l}} .
$$


With the use of Eq. (24) it can be rewritten in a more useful form:

$$
a=\frac{\sqrt{\rho_{l} \rho_{v}} a_{v} a_{l}}{\sqrt{\left(\rho_{l}\left(1-\alpha^{a}\right)+\rho_{v} \alpha^{a}\right)\left(p_{v} a_{v}^{2}\left(1-\alpha^{a}\right)+\alpha^{a} \rho_{l} a_{l}^{2}\right)}} .
$$

The minimum speed of sound in the mixture can now be deduced by solving $d a /\left.d \alpha^{a}\right|_{\alpha^{a}=\alpha_{\min }^{a}}=0$ and substituting $\alpha_{\min }^{a}$ back into Eq. (27). The $\alpha_{\min }^{a}$ is given by:

$$
\alpha_{\min }^{a}=\frac{\rho_{v}^{2} a_{v}^{2}-2 \rho_{v} \rho_{l} a_{v}^{2}+\rho_{l}^{2} a_{l}^{2}}{2\left(\rho_{l}-\rho_{v}\right)\left(\rho_{l} a_{l}^{2} \rho_{v} a_{v}^{2}\right)} .
$$

If $\rho_{l} \gg \rho_{v}$ than $\alpha_{\min }^{a} \rightarrow 1 / 2$ and the following expression for the minimal speed of sound is found

$$
a_{\min }=2 a_{v} \sqrt{\frac{\rho_{v}}{\rho_{l}}} .
$$

\section{References}

[1] Reynolds, O., 1886, "On the Theory of Lubrication and Its Application to Mr. Beauchamps Tower's Experiments, Including an Experimental Determination of the Viscosity of Olive Oil," Philos. Trans. R. Soc. London, 177, pp. 157234.

[2] Sun, D. C., and Chen, K. K., 1977, "First Effects of Stokes Roughness on Hydrodynamic Lubrication," J. Lubr. Technol., 99 pp. 2-9.

[3] Phan-Thien, N., 1981, "On the Effects of the Reynolds and Stokes Surface Roughnesses in a Two-Dimensional Slider Bearing," Proc. R. Soc. London, Ser. A, 377, pp. 349-362.

[4] Myllerup, C. M., and Hamrock, B. J., 1992, "Local Effects in Thin Film Lubrication," Proceedings of the $19^{\text {th }}$ Leeds Lyon conference on tribology.

[5] Myllerup, C. M., and Hamrock, B. J., 1994, "Perturbation Approach to Hydrodynamic Lubrication Theory," ASME J. Tribol., 116, pp. 110-118.
[6] Noordmans, J., 1996, "Solutions of Highly Anisotropic Stokes Equations for Lubrication Problems," ECCOMAS 96.

[7] Schäfer, C. T., Giese, P., and Woolley, N. H., 1999, "Elastohydrodynamically Lubricated Line Contact Based on the Navier-Stokes Equations," Proceedings of the $26^{\text {th }}$ Leeds Lyon conference on tribology.

[8] Odyck van, D. E. A., 2001. "Stokes Flow in Thin Films," Ph.D. thesis, University of Twente, The Netherlands.

[9] Odyck van, D. E. A., and Venner, C. H., 2003. "Stokes Flow in Thin Films," ASME J. Tribol., 125, pp. 1-14.

[10] Bair, S., Khonsari, M., and Winer, W. O., 1998, "High-Pressure Rheology of Lubricants and Limitations of the Reynolds Equation," Tribol. Int., 31)(10), pp. 573-586.

[11] Batchelor, G. K., 2000, An Introduction to Fluid Dynamics, Cambridge University Press, UK, ISBN 0521663962.

[12] Langlois, W. E., 1964, Slow Viscous Flow, The Macmillan Company, New York.

[13] Constantinescu, V. N., 1969, Gas Lubrication, ASME, New York.

[14] Dowson, D., and Taylor, C. M., 1979, "Cavitation in Bearings," Annu. Rev. Fluid Mech., 11, pp. 35-66.

[15] Elrod, H. G., 1981, “A Cavitation Algorithm,” ASME J. Lubr. Technol., 103, pp. 350-354.

[16] Delannoy, Y., and Kueny, J. L., 1990, “Two-Phase Flow Approach in Unsteady Cavitation Modeling," Cavitation and Multiphase Flow, 98, ASME FED, pp. $153-158$.

[17] Hoeijmakers, H. W. M., Janssens, M. E., and Kwan, W., 1998, "Numerical Simulation of Sheet Cavitation," Proceedings of the third international symposium on cavitation, Grenoble, France.

[18] Kubota, A., Kato, H., and Yamaguchi, H., 1992, "A New Modeling of Cavitating Flows: A Numerical Study of Unsteady Cavitation on a Hydrofoil Section," J. Fluid Mech., 240, pp. 59-96.

[19] Jakobsson, B., and Floberg, L., 1957, "The Finite Journal Bearing, Considering Vaporization,” Trans. Chalmers Univ. Tech., Göteborg, 190.

[20] Olsson, K., 1965, "Cavitation in Dynamically Loaded Bearings," Trans. Chalmers Univ. Tech. Göteborg, 308.

[21] van Wijngaarden, L., 1972, "One Dimensional Flow of Liquids Containing Small Gas Bubbles," Annu. Rev. Fluid Mech., 4, pp. 369-396. 